\title{
Heterogeneous Ignition of Coal Dust Clouds
}

\author{
F. J. HIGUERA A. LIÑÁN $\quad$ C. TREVIÑO*
}

\section{INTRODUCTION}

Two combustion modes exist in the burning of a cloud of coal or fuel particles in air when, as is usually the case, the reaction rates are highly sensitive to temperature. One of these is a slow kinetically controlled mode, and the other is a fast diffusion-controlled mode. The transition from the first mode to the second may occur in an abrupt way as an ignition event, or thermal explosion, if the conditions are supercritical, or also as a result of a flame propagation process.

In the classical theory of critical conditions for. thermal explosions, consumption of the reactant is neglected and then a steady state for the temperature is possible only for subcritical conditions. This state results from the competition between two processes: heat release by an exothermic strongly temperature-sensitive chemical reaction and heat loss from the region where the reaction takes place to the cold surroundings. The early works of Semenov and Frank-Kamenetskii were reviewed by Zel'dovich et al. [1]. In these works a conductive or conductive-convective heat loss was assumed. On the other hand, when there are particles suspended in a gas, as is the case in coal dust clouds burning in air, radiation becomes an important, often dominant, heat transfer mechanism, and the spatial derivatives of the radiative heat flux appear in the energy-balance equation. Evaluation of radiant heat loss requires, in principle, the calculation of the radiation intensity distribution from the equation of radiative transfer. Even for a one-dimensional medium in local thermodynamic equilibrium, this is an integrodifferential equation strongly coupled with the en- 
ergy-balance equation through the dependence of the blackbody intensity on the local temperature. The resulting nonlinear, nonlocal problem is a highly complicated one, and several approximating techniques, including alternative expressions for the radiative heat transfer, have been developed to obtain approximate results. One of these techniques, the differential approximation, will be used in this paper. It is based on certain averages on the transfer equation leading, after definite approximations, to simple differential equations for the first moments of the intensity distribution. Details on the existing alternative formal methods to obtain the differential approximation can be found in Refs. 2 and 3. Directional averaging of the intensity distribution is involved in the differential approximation, so that it is better suited for planar geometries, and it has found wide application in dusty flame models, where, in addition, it reduces to the exact results in the optically thin and optically thick limits (Planck and Rosseland approximations, respectively).

Models of dust-air flame propagation including radiation have been proposed. Differing in other respects, the models of Essenhigh and Csaba [4] and Bhaduri and Bandyopadhyay [5] both treat the flame as a gray body of fixed temperature; the radiation intensity decays exponentially away from the flame due to the absorption by the particles, but radiation emitted or scattered by these particles is neglected. The differential approximation to model radiative transfer has been used by Ozenova and Stepanov [6] and Krazinski et al. [7]. Field et al. [8] employed flux methods to determine radiation fluxes in scattering reacting media. The radiative equation, together with the nonlinear stationary energy-balance equation, was integrated numerically by Khalil et al. $[9,10]$ in the analysis of reaction-radiation equilibria of particle suspensions in plane geometry with a single-temperature model; the effects of the radiative properties of the medium and the walls, and of the parameters of the heat generation model on the critical conditions for extinction, are illustrated by numerical examples. The ignition problem was solved by Joulin [11] using the differential approximation. His results show that reaction-radiation equilibria represent a transition path from the Semenov to the FrankKamenetskii critical conditions, which are recov- ered for optically thin and optically thick media, respectively.

The ignition process can be the result of reactions occurring in the gas phase due to the released volatiles or can result from reactions at the surface of the particles. What actually happens in a given case seems to depend on many factors, including heat-up rate, particle size, and gas-phase composition. The older theories were based on gas-phase ignition, but experimental results exist that favor the view of heterogeneous ignition, the pyrolysis of the solid taking place well before or well after the ignition event. An extensive review on the relevance of heterogeneous ignition and the nature of the heterogeneous reactions was presented by Essenhigh [12]. The effects of surface processes (adsorption, chemical reaction, desorption) are often accounted for by a proper choice of the order and activation energy of an overall Arrhenius reaction. A model including two heterogeneous reactions was applied by Libby and Blake $[13,14]$ to the theoretical study of the ignition, combustion, and extinction of a single carbon particle. On the other hand, it is recognized that the heterogeneous reactions can be diffusion controlled during most of the particle lifetime, especially for large particles or high temperatures. In fact, Libby and Blake [13] showed that the consumption rate does not change very much when the state of the gas phase goes from chemically frozen to chemical equilibrium.

In this paper a heterogeneous ignition analysis is presented for a coal particle suspension. Only the heterogeneous reaction $\mathrm{C}(\mathrm{s})+1 / 2 \mathrm{O}_{2} \rightarrow \mathrm{CO}$ is considered, and the devolatilization and gas-phase chemical reactions are neglected. Radiative transfer is modeled by means of the differential approximation. We shall account in the analysis for the temperature differences between the particles and the ambient gas, which are important when the thermal capacity of the gas is large compared with the thermal capacity of the particles.

\section{FORMULATION}

We consider the following physical problem. A cloud of spherical monodisperse carbon particles at temperature $T_{s 0}$ is suddenly injected in the space 
between two parallel infinite planar walls. This space is filled with a hot oxidizing gas initially at temperature $T_{g 0}$ and oxygen mass fraction $Y_{g 0}$. The walls themselves are assumed to be at a constant temperature $T_{w}$. Energy transfer by radiation between the particles and between the particles and the walls is accounted for in the analysis, using the Eddington differential approximation. In addition, conductive heat transfer between the particles and the gas is included. The gas is assumed to be transparent to the radiation. For the ignition analysis, only the direct reaction $\mathrm{C}(\mathrm{s})+1 / 2 \mathrm{O}_{2} \rightarrow \mathrm{CO}$ is considered, with a mass consumption rate of carbon per unit surface given by the Arrhenius law

$$
m^{\prime \prime}=Z Y \exp \left(-T_{a} / T_{s}\right)
$$

where $Z$ represents the preexponential factor, $T_{a}$ is the activation temperature, $T_{s}$ is the temperature of the solid particle surface, and $Y$ is the mass fraction of oxygen at the particle surface.

Due to the small gas/solid density ratio, the thermal diffusivity of the gas is much larger than that of the solid, and the heat conduction in the gas phase around every particle is a quasi-steady process. Besides, in most cases, the thermal conductivity of the solid is much larger than that of the gas, and therefore the temperature of each particle is nearly uniform during the particle heating. Under these conditions, the governing equations and boundary conditions for the problem are given by

$$
\begin{aligned}
& \frac{4}{3} \pi R^{3} n \rho_{s} C_{s} \frac{\partial T_{s}}{\partial t}=-4 \pi R n k_{g}\left(T_{s}-T_{g}\right) \\
& -\frac{\partial q}{\partial x}+4 \pi R^{2} n Q m^{\prime \prime} \\
& L \frac{\partial}{\partial x}\left(L \frac{\partial q}{\partial x}\right)=3 q+L \frac{\partial}{\partial x}\left(4 \sigma T_{s}^{4}\right) \\
& x= \pm l: \quad L \frac{\partial q}{\partial x} \pm 2 q-4 \sigma T_{s}^{4}=-4 \sigma T_{w}{ }^{4} \\
& \rho_{g} C_{p} \frac{\partial T_{g}}{\partial t}=-4 \pi R n k_{g}\left(T_{g}-T_{s}\right) \\
& \rho_{g} \frac{\partial Y_{g}}{\partial t}=-4 \pi R n \rho_{g} D_{g}\left(Y_{g}-Y\right)
\end{aligned}
$$

$\rho_{g} D_{g} \frac{Y_{g}-Y}{R}=\left(\frac{4}{3}+Y_{g}\right) m^{\prime \prime}$

$\rho_{s} \frac{\partial R}{\partial t}=-m^{\prime \prime}$

and the initial conditions are the following:

$$
\begin{gathered}
t=0: \quad T_{s}=T_{s 0}, \quad T_{g}=T_{g 0}, \\
Y_{g}=Y_{g 0}, \quad R=R_{0}
\end{gathered}
$$

Equation 2 is an energy balance for the particles. $R$ is the particle radius; $n$ is the particle number density; $\rho_{s}$ and $C_{s}$ are the density and specific heat, respectively, of the solid phase; and $T_{g}$ and $k_{g}$ are the temperature and thermal conductivity, respectively, of the gas. $Q$ is the heat released per unit mass of carbon, and $x$ is the distance normal to the walls, measured from the center of the slab; the thickness of the slab is $2 l$. Equation 3a provides the link between the radiation flux $q$ and the particle temperature. As it stands, this equation models a gray nonscattering medium, but generalizations to include more complex optical behaviors are straightforward within the framework of the differential approximation. $\sigma$ is the Stefan-Boltzmann constant, and $L$ is the optical length, given by $L=1 / n \pi R^{2}$, except for an efficiency factor of order unity. Note that the temperature of the particles near the walls is different from the wall temperature. The boundary condition, Eq. 3b, corresponds to walls behaving as black bodies. Relations 4-6 result from the analysis of the quasisteady response of the gas phase (see, for example, Libby and Blake [13]). They have been written in a form valid for $m^{\prime \prime} R C_{p} / k_{g} \ll 1$, which is justified for the ignition analysis. $D_{g}$ denotes the oxygen diffusion coefficient in the gas phase.

When studying the ignition problem in the limit of large Zel'dovich numbers, the time variations of the particle radius and mass concentration of oxygen can be neglected in a first approximation. In addition to this, the oxygen mass concentration at the surface of the particles is the same as in the gas phase. Therefore Eqs. 5-7 do not play any role in what follows. It is clear that these simplifications eliminate the fast-burning regimes appearing in the analysis of Khalil et al. [9], in which the chemical reaction is diffusion-controlled. As 
pointed out by Joulin [11], the critical conditions presented by these regimes correspond to quenching, and, though important, they bear no relation to the ignition analysis addressed here.

To reduce the equations to nondimensional form, we introduce the following dimensionless variables:

$$
\begin{array}{rlrl}
\theta_{s}=T_{s} / T_{\mathrm{g} 0}, & \theta_{\mathrm{g}} & =T_{\mathrm{g}} / T_{\mathrm{g} 0}, \quad \phi=q / q_{c}, \\
\tau=t / t_{c}, & \xi=\sqrt{3} x / L_{0}
\end{array}
$$

where

$t_{c}=\rho_{s} C_{s} R_{0}^{2} / 3 k_{g}$

$q_{c}=\frac{4}{\sqrt{3}} \pi R_{0} n L_{0} k_{g} T_{g 0} \quad$ with $L_{0}=\frac{1}{n \pi R_{0}^{2}}$ by

The resulting nondimensional problem is given

$\frac{\partial \theta_{s}}{\partial \tau}=-\left(\theta_{s}-\theta_{g}\right)-\frac{\partial \phi}{\partial \xi}+\Delta \exp \left(\beta \frac{\theta_{s}-1}{\theta_{s}}\right)$

$\frac{\partial^{2} \phi}{\partial \xi^{2}}=\phi+\frac{1}{N} \frac{\partial \theta_{s}^{4}}{\partial \xi}$

$\xi= \pm X: \quad \frac{\partial \phi}{\partial \xi} \pm \frac{2}{\sqrt{3}} \phi-\frac{\theta^{4}}{N}=-\frac{\theta_{w}^{4}}{N}$

$I \frac{\partial \theta_{g}}{\partial \tau}=-\left(\theta_{g}-\theta_{s}\right)$

$\tau=0: \quad \theta_{s}=\theta_{s 0}, \quad \theta_{g}=1$

where

$\Delta=\frac{Q Y_{g 0}}{C_{p} T_{g 0}} \frac{Z C_{p} R_{0}}{k_{g}} \exp (-\beta), \quad \beta=\frac{T_{a}}{T_{g 0}}$,

$I=\frac{\rho_{g} C_{p}}{(4 / 3) \pi R_{0}^{3} n \rho_{s} C_{s}}$,

$X=\frac{\sqrt{3} l}{L_{0}}, \quad \theta_{w}=\frac{T_{w}}{T_{g 0}}, \quad N=\frac{k_{g}}{\sigma R_{0} T_{g 0}{ }^{3}}$

Here $\Delta$ is the Damköhler number; the parameter $I$ represents the gas/particles thermal capacity ratio;
$X$ is the nondimensional thickness of the slab, referred to the absorption length; $N$ is a conduction/radiation parameter and represents the ratio of heat transferred by conduction to that transferred by radiation; this parameter is of order unity for particles of $50 \mu \mathrm{m}$ radius and a gas temperature of $1000 \mathrm{~K}$.

The situation that we envisage here is that of an initially cold particle cloud $\left(\theta_{s 0}<1\right)$ heated by conduction from the hot gas and by radiation from the walls, if the walls are also hot.

\section{IGNITION CONDITIONS}

The nondimensional activation energy is, in our case, a large number $(\beta \gg 1)$ so it makes sense to define a critical temperature $\theta_{l}$ such that

$\Delta \exp \left[\frac{\beta\left(\theta_{I}-1\right)}{\theta_{I}}\right]=1$

For values of the particle nondimensional temperature $\theta_{s}(\xi, \tau)$ smaller than $\theta_{I}$, the heat release due to the chemical reaction in Eq. 11 is exponentially small and does not play any role. In this frozen evolution, $\theta_{s}(\xi, \tau)$ increases from its initial value $\theta_{s 0}$ and the nondimensional gas temperature $\theta_{g}(\xi$, $\tau)$ also departs from unity. Both temperatures would tend to reach asymptotically the final common value

$\theta_{s}=\theta_{g}=\theta_{w}$

in the absence of heat release by the chemical reaction. Ignition will occur, however, when the frozen particle temperature first reaches the value $\theta_{I}$ at some point. By calling $\theta_{M}$ the maximum value of the particle temperature in the frozen evolution, the criterion for ignition to occur can be written as $\theta_{M}>\theta_{I}$, and the ignition time is easily determined, in the limit $\beta \rightarrow \infty$, from the frozen temperature distribution. When $\theta_{M}=\theta_{w}$, the maximum temperature is reached asymptotically for large values of $\tau$. In this case it is possible to look for the critical conditions for ignition applying a quasi-steady treatment, that is, determining whether or not a balance can be attained between the heat release by the reaction and the heat 
transfer to the walls. The problem given by Eqs. 11-13 with the time derivatives removed has been solved by Joulin [11]. For numerical work not relying on the Eddington approximation, see Khalil et al. $[9,10]$.

Another situation in which a modified form of the quasi-steady treatment of the thermal ignition is applicable will be considered here. It corresponds to the realistic limit $I \gg 1$, in which the thermal capacity of the solid particles is much smaller than that of the gas, and includes in particular the case of near-stoichiometric mixtures of coal particles and air. In this case there are two stages in the frozen evolution of $\theta_{s}$. In the first stage $[\tau=O(1)], \theta_{s}(\xi, \tau)$ increases without any appreciable change in $\theta_{g}$ and tends to an asymptotic distribution with the maximum value at the center of the $\operatorname{slab}(\xi=0)$ if $\theta_{w}<1$, or at the walls $(|\xi|=X)$ if $\theta_{w}>1$. In the second stage [ $\tau=$ $O(I)]$, the gas temperature $\theta_{g}(\xi, \tau)$ and the temperature of the particles change due to the radiative heat exchange with the walls. If $\theta_{w}<1$, the temperatures of both phases decrease during this stage, and the maximum value at the end of the first stage can be identified with $\theta_{M}$. In the opposite case, $\theta_{w}>1$, both temperatures increase in the second stage toward the final common value $\theta_{w}$, and the appropriate criticality analysis is that of Joulin [11].

CASE 1. $\left|1-\theta_{w}\right|=O(1)$

The particle temperature distribution at the end of the first stage is given by the solution of Eqs. 11 and 12 without the time derivative term and with $\theta_{g}$ $=1$. The frozen distribution $\theta_{F}(\xi)$ results when, in addition, the reaction term is removed from Eq. 11. The maximum particle temperature obtained from this frozen solution is plotted in Fig. 1 as a function of $\theta_{w}$ for several values of $X$ and $N$. The frozen temperature near the center of the slab $(\xi \ll$ 1) is $\theta_{F} \approx \theta_{M}-\theta_{2} \xi^{2}$, with

$\theta_{2}=\left(1-\theta_{M}\right) / 2\left(1+12 \theta_{M}^{2} / N\right)$.

In order to account for the effect of the chemical reaction, and to determine the critical conditions

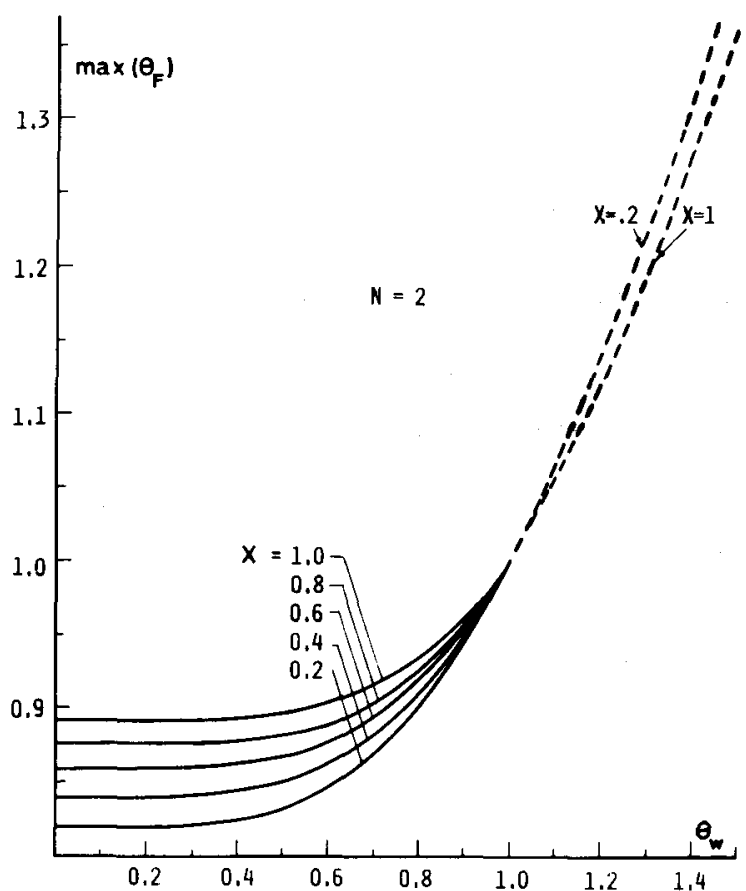

(a)

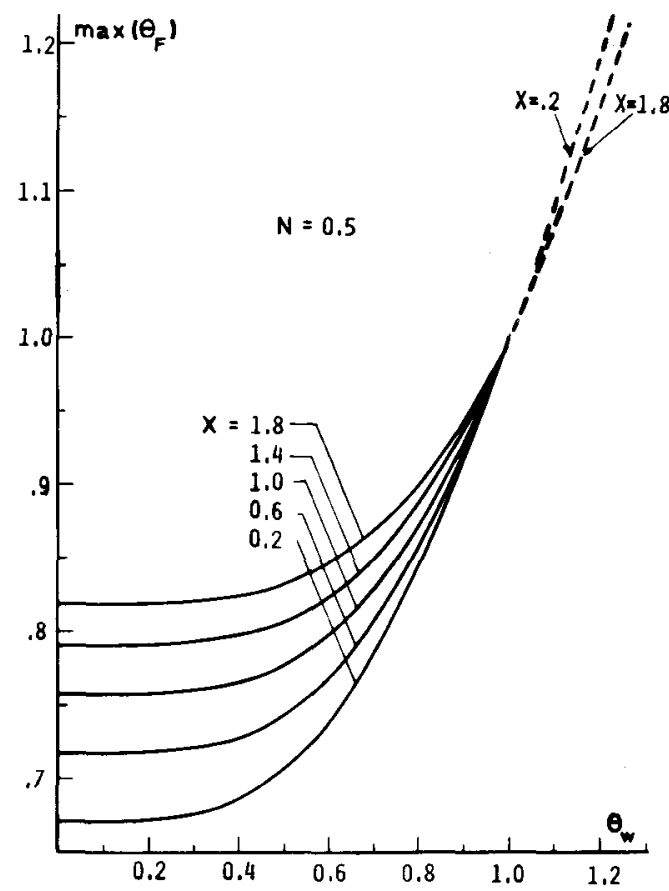

(b)

Fig. 1. Maximum value of $\theta_{F}(\xi)$ as a function of $\theta_{w}$. The maximum is attained at the center of the slab for $\theta_{w}<1$ (continuous lines) and at the walls for $\theta_{w}>1$ (dashed lines). 
for ignition in the case $\theta_{w}<1$, the variables

$$
\begin{gathered}
\tilde{\theta}=\beta\left(\theta_{s}-\theta_{F}\right), \quad \tilde{\phi}=\frac{\beta^{3 / 2} N \theta_{2}^{1 / 2}}{4 \theta_{M}{ }^{3}}\left(\phi-\phi_{F}\right), \\
\eta=\beta^{1 / 2} \theta_{2}^{1 / 2} \xi
\end{gathered}
$$

are introduced. Here $\phi_{F}$ is the nondimensional radiation flux in the absence of reaction, and $\eta$ is of order unity in the narrow region around the middle point where the temperature is higher and the reaction rate is appreciable. When the variables in Eq. 18 are carried to Eq. 12a, there results, in first approximation,

$\frac{d^{2} \tilde{\phi}}{d \eta^{2}}=\frac{d \tilde{\theta}}{d \eta}$,

reflecting the fact that the reaction region is optically thin. Equation 19 can be integrated once, and the integration constant vanishes, as can be seen from the matching with the outer reactionfree region where $\xi=O(1)$. Using this result together with Eq. 18 in the simplified form of Eq. 11 , one obtains

$\delta \exp \left(\frac{\tilde{\theta}-\eta^{2}}{\theta_{M}}\right)-\left(1+\frac{4 \theta_{M}^{3}}{N}\right) \tilde{\theta}=0$

where $\delta=\beta \Delta \exp \left[\beta\left(\theta_{M}-1\right) / \theta_{M}\right]$. This is a Semenov-like problem, with $\eta$ playing the role of a parameter. The ignition occurs for $\eta=0$, and the critical value of $\delta$, which will be denoted by $\delta_{c}$, is given by

$\delta_{c}=\theta_{M}^{2} \frac{1+4 \theta_{M}^{3} / N}{e}$

where $\theta_{M}$ in Eq. 21 and in the definition of $\delta$ should be taken from Fig. 1 .

It must be remarked that small changes in particle radius (see Eq. 7) and optical length should have been included in the determination of the critical conditions. In addition, the radial flux due to the burning modifies the particle-gas heat transfer rate, and the first term on the right-hand side of Eq. 2 should have been modified accordingly. When accounting for such effects, new terms appear in the energy balance, Eq. 20; however, as can be easily verified, these terms are proportional to $C_{p} T_{g 0} / Q$, which is a small number, $C_{p} T_{g 0} / Q \approx 0.13$ at $T_{g 0}=1000 \mathrm{~K}$, so it seems safe to neglect them. A second remark is that the rather strong assumption $I / \beta \gg 1$ has been made in order to neglect temperature changes in the gas up to the orders involved in the analysis. When this assumption is released, and the small terms commented on before are included, one is led to a transient ignition analysis. Results of this analysis will be presented elsewhere. (A limiting case is analyzed in Appendix A.)

In the case $\theta_{w}>1$, ignition can occur in the second stage, during the heating of the gas phase. However, as this is a slow process, it could be of interest to determine the critical conditions for ignition to occur in the first stage. The maximum particle temperature is attained in this case near the hot walls, and this is the place where the reaction is first appreciable. It turns out that the result of Eq. 21 is still valid, with the same remarks as before, but $\theta_{M}$ must be replaced everywhere by the value obtained from the dashed part of the curves in Fig. 1.

CASE 2. $\left|1-\theta_{w}\right|=O(1 / \beta)$

In this case the reaction is important in the entire space between the walls, and a separate analysis is necessary. When looking for quasi-steady states and critical conditions, it is convenient to introduce the variables

$\theta=\beta\left(\theta_{s}-1\right), \quad \Phi=\beta \phi$

in Eqs. 11 and 12. Linearizing the exponent in the reaction term in Eq. 11, and also the fourth-power terms in Eqs. 12a and $12 \mathrm{~b}$, there results the simplified problem

$\frac{d \Phi}{d \xi}=\Delta \exp (\theta)-\theta$,

$\frac{d^{2} \Phi}{d \xi^{2}}=\Phi+\frac{4}{N} \frac{d \Theta}{d \xi}$,

$\xi= \pm X: \quad \frac{d \Phi}{d \xi} \pm \frac{2}{\sqrt{3}} \Phi-\frac{4 \Theta}{N}=-\frac{4 \Theta_{w}}{N}$, 
where

$\theta_{w}=\beta\left(\theta_{w}-1\right)$.

Equation 23 represents a balance between the heat released by the chemical reaction and that transferred by radiation between the particles and the walls, and by conduction between the particles and the gas.

\section{Asymptotic Limits}

Let us begin by considering the limit of the large conduction/radiation parameter $(N \gg 1)$. In this case the radiation flux is small, $\Phi=O(1 / N)$ and can be neglected in Eq. 23. The resulting Semenov problem leads to the critical Damköhler number $\Delta_{c}$ $=1 / e$ and to the uniform temperature $\theta=1$ at criticality. Looking now for the small effect of the radiation flux, the result is the corrected condition

$$
\Delta_{c}=\left\{\begin{array}{c}
\frac{1}{e}\left[1+\frac{8\left(1-\theta_{w}\right) / N}{(1+2 / \sqrt{3}) e^{X}+(1-2 / \sqrt{3}) e^{-X}}\right] \\
\text { for } \theta_{w}<1 \\
\frac{1}{e}\left[\begin{array}{c}
\left.1-\frac{4\left(\theta_{w}-1\right)\left(e^{X}+e^{-X}\right) / N}{(1+2 / \sqrt{3}) e^{X}+(1-2 / \sqrt{3}) e^{-X}}\right] \\
\text { for } \theta_{w}>1,
\end{array}\right.
\end{array}\right.
$$

which is uniformly valid in $X$. Steady states exist for $\Delta<\Delta_{c}$, whereas for $\left.\Delta\right\rangle \Delta_{c}$ vigorous burning is to be expected.

In the opposite limit, $N \ll 1$ heat exchange with the gas becomes negligible, and one recovers the problem analyzed by Joulin [11], after an appropriate redefinition of the variables, which includes linearization around the wall temperature instead of the temperature of the gas. This can be seen best by using the new variables $\Phi^{\prime}=N \Phi, \theta^{\prime}=\theta-$ $\theta_{w}$, and $\Delta^{\prime}=N \Delta \exp \left(\Theta_{w}\right)$, and then letting $N \rightarrow$ 0 .

For optically thin media, $X \ll 1$, Eqs. 24 and 25 lead to $d \Phi / d \xi=4\left(\theta-\theta_{w}\right) / N$, and when this is carried to Eq. 23 a Semenov-like problem results. The critical Damköhler number, including the second term in an expansion for small values of $X$, is given by

$$
\begin{aligned}
\Delta_{c}= & \frac{\exp \left[-4 \Theta_{w} /(4+N)\right]}{e N} \\
& \times\left[4+N+\frac{8 X}{\sqrt{3}}\left(\frac{N \Theta_{w}}{4+N}-1\right)+o(X)\right]
\end{aligned}
$$

On the other hand, for optically thick media, $X$ $\Rightarrow 1$, radiation is equivalent to heat conduction in most of the space. The resulting radiation flux is too small to appear in the energy balance (Eq. 23) in first approximation, and a Semenov problem results once again, which would lead to $\Delta_{c}=1 / e$. However, the resulting temperature field (given by $\theta=1$ at critical conditions), does not satisfy the boundary condition (Eq. 25), and narrow regions appear near the walls where the optically thick approximation can no longer be applied. In the narrow region near the left wall (the other is identical), the problem to be solved is given by Eqs. 23 and 24 subject to the boundary conditions

$$
\begin{array}{ll}
\xi=0: & \frac{d \Phi}{d \xi}-\frac{2}{\sqrt{3}} \Phi-\frac{4 \Theta}{N}=-\frac{4 \Theta_{w}}{N} \\
\xi \rightarrow \infty: \quad & \Theta=\theta_{\text {outer }},
\end{array}
$$

where $\xi$ is now measured from the wall. The second boundary condition comes from the matching with the outer region; $\theta_{\text {outer }}$ is the (smaller) root of $\Delta \exp (\theta)-\theta=0$. Solutions for this inner problem cease to exist for $\Delta>\Delta^{*}\left(\Theta_{w}, N\right)$ $<1 / e$, and when the inequality holds the critical Damköhler number is determined by the presence of the narrow regions. This is the case for $\theta_{w}>1$, as will be shown later.

Another distinguished limit in which the radiation flux is important all over the domain, even though $X \gg 1$, will be analyzed in Appendix B.

\section{General Solution and Discussion}

To obtain the solution of Eqs. 23-25 and the critical conditions for ignition in a general case, it is convenient to eliminate $\xi$ by using the fact that the differential equations 23 and 24 are autonomous. Equation 24 can be written in terms of $\Phi$ 
and $\theta$ with the help of Eq. 23, and it takes the form

$$
\left(\Delta e^{\theta}-\theta\right)\left(\Delta e^{\theta}-1-\frac{4}{N}\right) \frac{d \theta}{d \Phi}=\Phi,
$$

which can be integrated to give

$$
\begin{aligned}
& \Delta^{2} e^{2 \theta}-2\left(\theta+\frac{4}{N}\right) \Delta e^{\theta} \\
& +\left(1+\frac{4}{N}\right) \theta^{2}=\Phi^{2}-A,
\end{aligned}
$$

where $A$ is a constant. At the center of the slab, the radiation flux $\Phi$ is zero, due to the symmetry of the problem, and Eq. 31 can be used to relate $A$ to the central temperature. Equation 31 has been plotted schematically in Fig. 2 for different (increasing) values of $A$. In the case $\Delta<1 / e$, a phase portrait looking like the mirror image of that plotted in Fig. 2 can also be obtained. In any case, there exist orbits with cusps at $\Phi=0$ and the values of $\theta$ indicated in the caption to Fig. 2 for well-defined values of $A$.

The relation between $\xi$ and the temperature is given by

$\frac{d \xi}{d \theta}=\frac{\Delta e^{\theta}-1-4 / N}{\Phi}$

with $\Phi(\theta)$ taken from Eq. 31. Equation 32 is the result of eliminating $d^{2} \Phi / d \xi^{2}$ between Eq. 24 and the derivative of $\mathrm{Eq} .23$.

The boundary condition Eq. 25 can also be transformed to

$\Delta e^{\theta_{e}}-\theta_{e}+\frac{2}{\sqrt{3}} \Phi_{e}=\frac{\theta_{e}-\theta_{w}}{N / 4}$,

which has been specialized to the right wall. $\theta_{e}$ and $\Phi_{e}$ are the unknown values of the particle temperature and radiation flux at the wall.

The solution procedure is as follows: Fixed values are given to $\Delta, N$, and $\theta_{w}$, and the temperature of the particles at the wall $\theta_{e}$ is used as a parameter. The flux $\Phi_{e}$ is obtained from Eq. 33, and the constant $A$ then results from Eq. 31 . This allows one to determine which of the possibil-
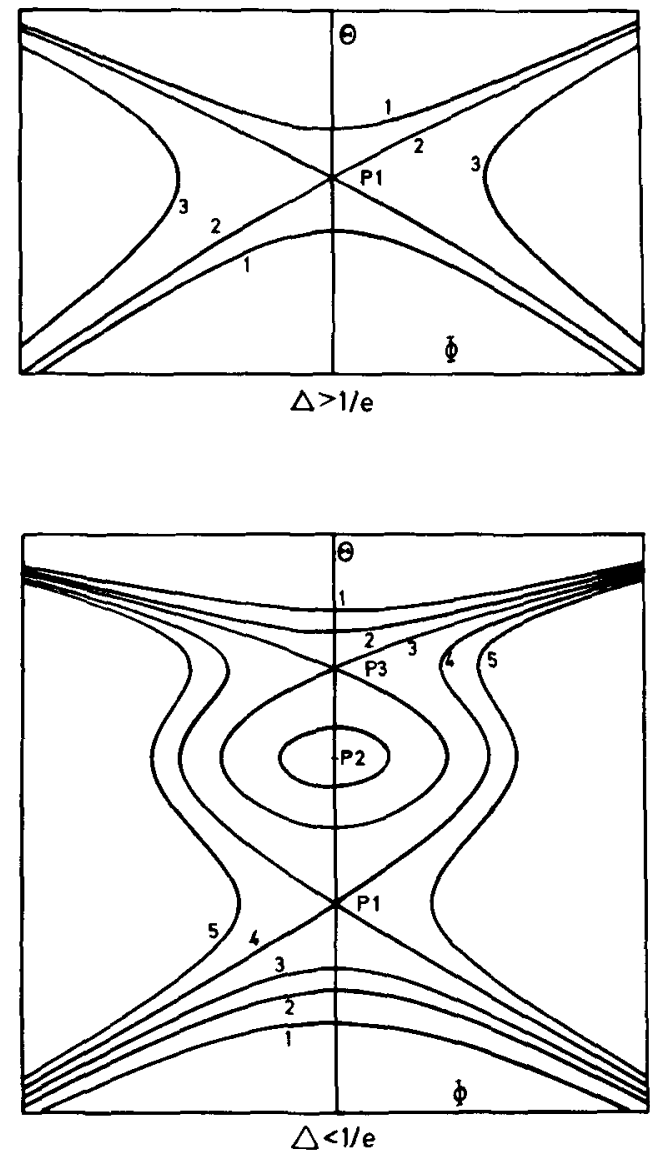

Fig. 2. Phase portrait for Eq. 30. The curves labeled with increasing numbers correspond to increasing values of $A$ in Eq. 31. For $\Delta>1 / e$ the temperature at $\mathrm{P} 1$ is $\ln [(4+N) / N \Delta]$. For $\Delta<1 / e$ the temperature at $P 1$ is the lower root of $\Delta$ $\exp (\theta)-\theta=0$; at $\mathrm{P} 2$ and $\mathrm{P} 3$ the temperatures are given by the upper root of this equation and the value $\ln [(4+N) / N \Delta]$, but their order depends on the values of $\Delta$ and $N$.

ities shown in Fig. 2 is actually relevant, and, in particular, the value(s) of the temperature at the center of the slab can be obtained by solving, iteratively, Eq. 31 with $\Phi=0$. Finally, $X$ results from Eq. 32 through a quadrature. Critical conditions are defined as those leading to maxima or minima of the function $X\left(\theta_{e}\right)$ thus determined. The critical Damköhler number as a function of $X$ for several values of $N$ and two different values of $\boldsymbol{\theta}_{w}$ is plotted in Fig. 3. Solutions exist for $\Delta<$ $\Delta_{c}\left(X, N, \Theta_{w}\right)$.

For $\theta_{w}=0$ (Fig. 3a), the maximum tempera- 
ture occurs at the center of the slab, and the critical conditions reflect the well-known fact that for a given $X$ the heat released by the reaction can be evacuated steadily only if it is smaller than a critical value, which decreases when $X$ increases. This is a general feature for $\theta_{w}<1$. Sketches of the temperature and radiation flux under critical and subcritical conditions are given in Fig. 4a.

The results for $\theta_{w}=2$ (Fig. 3b) reflect a different behavior. At least on part of the curves, $\Delta_{c}$ increases with $X$ and is smaller than $1 / e$. The temperature in this range is minimum at the center of the slab, and the right-hand side of Eq. 23, which represents the combined effect of heat release by the reaction and heat exchange with the gas, is negative there, corresponding to an " effective" endothermic reaction. The effective heat release can become positive at some distance from the center, reaching a maximum value at the walls, where ignition will occur if the heat loss toward the center is high enough, that is, if $X$ is small (see Fig. 4c). This may be an appropriate explanation for the rightmost part of the curves in Fig. 3b, when $X$ is large enough or $N$ small enough. However, for smaller values of $X$ and larger values of $N$, the effective heat release, on the right-hand side of Eq. 23, is seen to be always negative (Fig. 4b). Even though such a system would clearly not present ignition if the heat were evacuated by the usual conduction, the situation changes when radiation is involved. Rewriting Eq. 32 in the alternative form

$\Phi=-\left(1+4 / N-\Delta e^{\Theta}\right) \frac{d \Theta}{d \xi}$,

it can be seen [11] that in the present problem also the heat flux is proportional to the temperature gradient but the apparent conductivity depends strongly on the temperature and can be zero or

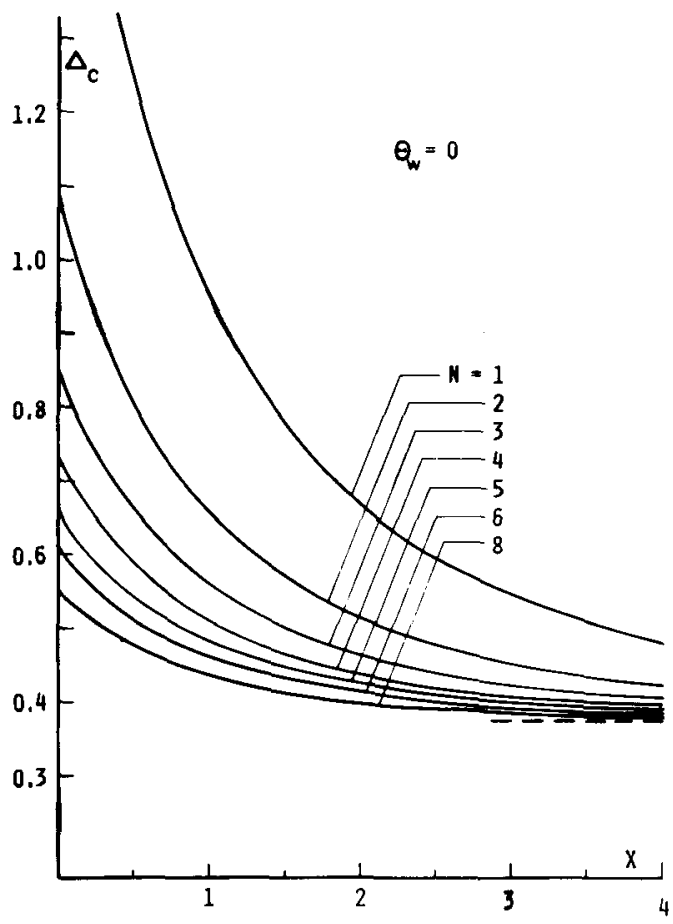

(a)

Fig. 3. Critical Damköhler number as a function of the thickness of the slab for several values of the conduction/radiation parameter. The curves are monotonic when $\theta_{w}<1$ and can present a minimum when $\theta_{w}>1$ if $N$ is sufficiently small. 


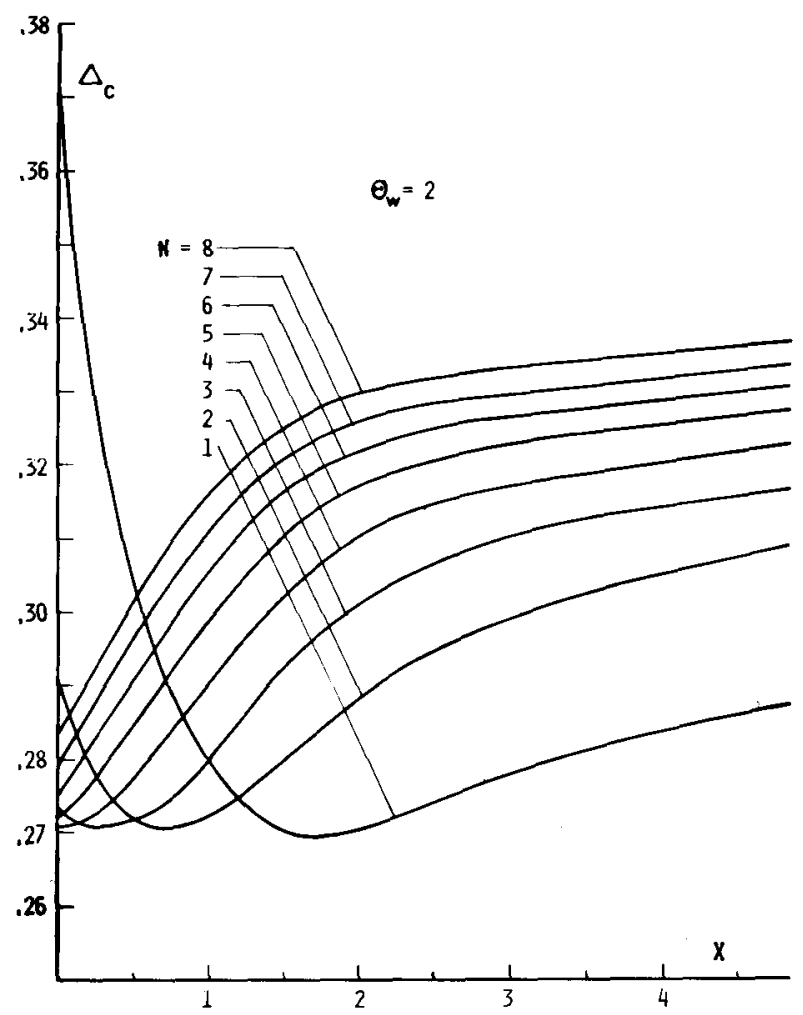

(b)

Fig. 3. (Continued).

negative. Small values of $\left(1+4 / N-\Delta e^{\theta}\right)$ are seen to occur near the walls. Let us consider the extreme case in which this is zero at some point between the plates; there, $d \Phi / d \xi=\Delta e^{\theta}(d \theta /$ $d \xi)^{2} \geqslant 0$, whereas $d \Phi / d \xi<0$ is required in Eq. 23. The need to avoid this paradox explains the presence of critical conditions for the existence of steady solutions even in this case.

For $N<4$, the function $\Delta_{c}(X)$ plotted in Fig. $3 \mathrm{~b}$ decreases in a range of $X$. The temperature distribution corresponding to this range is similar to that of the case $\theta_{w}=0$, presenting a maximum at the center of the slab (see Fig. 4a). This is a general feature for $\theta_{w}>1$, the range of decreasing $\Delta_{c}$ appearing for small values of $X$ when $N$ becomes smaller than $4 /\left(\theta_{w}-1\right)$, as can be inferred from the asymptotic expression, Eq. 28. When $\epsilon=4 /\left(\theta_{w}-1\right)-N \ll 1$, the critical Damköhler number $\Delta_{c}(X)$ for $X=\epsilon X, x=$ $O(1)$, is given by the expansion

$\Delta_{c}=\Delta_{0}+\epsilon^{2} \Delta_{2}+\cdots$, where

$$
\begin{aligned}
\Delta_{0} & =\frac{\theta_{w}}{\exp \theta_{w}}, \quad \Delta_{2}=\left(\theta_{w}-1\right)^{2}\left[\frac{2 x}{\sqrt{3}}\right. \\
& \left.-\theta_{w}+1\right]^{2} \frac{\exp \theta_{w}}{2 \theta_{w}^{2}} .
\end{aligned}
$$

The temperature distribution at critical conditions takes the form

$\boldsymbol{\theta}=\boldsymbol{\theta}_{w}+\epsilon \Theta_{1}+\epsilon^{2} \Theta_{2}+\cdots$,

where

$$
\begin{array}{r}
\theta_{1}=-\frac{\theta_{w}-1}{\theta_{w}}\left[\frac{2 x}{\sqrt{3}}-\theta_{w}+1\right], \\
\theta_{2}=-\frac{\theta_{1}}{\theta_{w}-1} \frac{(\xi / \epsilon)^{2}-1}{2}+C .
\end{array}
$$

The minimum value of $\Delta_{c}$ is attained for $x=$ $\sqrt{3}\left(\theta_{w}-1\right) / 2$. Note that the parabolic tempera- 
ture distribution changes from convex to concave when $x$ crosses the value corresponding to the minimum. The constant $C$ in Eq. 37 is a complicated expression that does not depend on $\xi$.

The range of values of $X$ on which $\Delta_{c}(X)$ decreases widens when $N$ decreases, in agreement

(a)

(b)

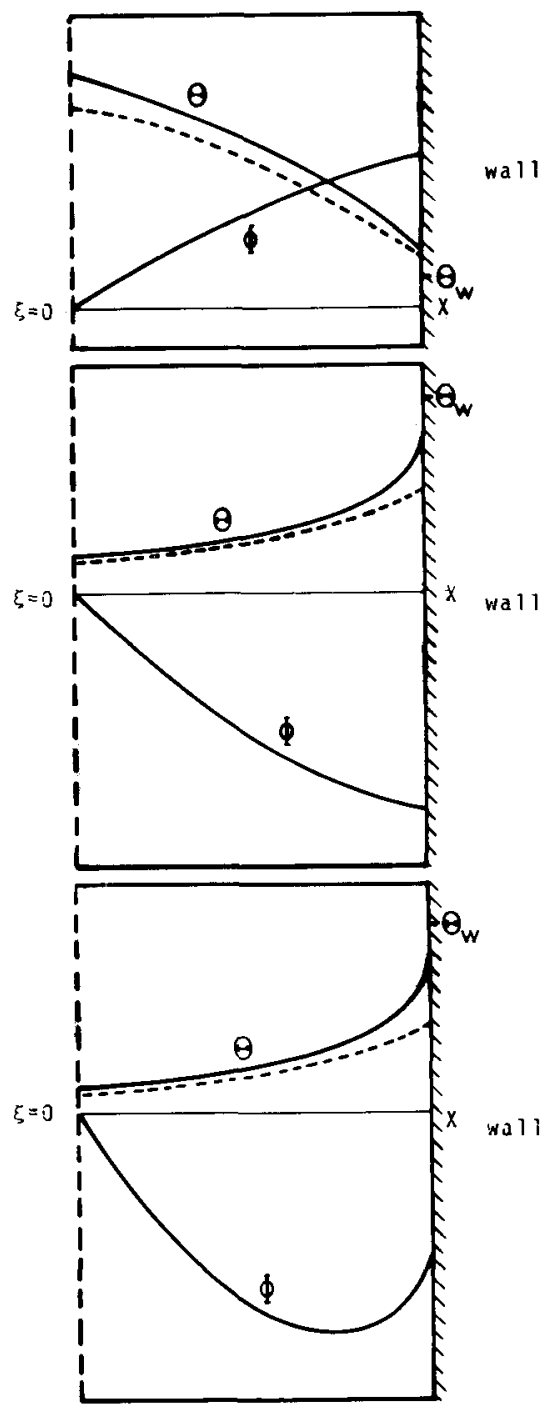

Fig. 4. Sketches of the temperature and radiation flux profiles at critical conditions (solid lines) and slightly subcritical conditions (dashed lines). (a) The maximum temperature appears at the center and develops a cusp there under critical conditions. (b) Wholly endothermic "effective" reaction. (c) Reaction effectively endothermic near the center and exothermic near the wall. In both cases (b) and (c) the temperature slope is infinite at the wall under critical conditions. with what should be expected from the asymptotic behavior for $N \ll 1$. (See Ref. 11.)

\section{Solution for an Infinite Domain}

Finally, let us come back to the solution of the problem presented by Eqs. 23, 24, and 29, which provides, in particular, the values of the asymptotes of the curves in Fig. 3 for $X \rightarrow \infty$. The procedure applied for finite values of $X$ can also be used now with minor changes. For given values of $\Delta$ and $N$, the constant $A$ results from Eq. 31 particularized at infinity, where $\Phi=0$ and $\theta$ is given by the second boundary condition, Eq. 29 . This temperature corresponds to the point $\mathrm{P} 1$ in Fig. $2 b$ and leads to a divergent integral in Eq. 32, as it should. Applying Eq. 31 at the wall, the radiation heat flux there can be obtained as a function of the local particle temperature $\theta_{e}$, which will be taken as a parameter, in the same way as before. Finally the wall temperature $\theta_{w}$ as a function of $\theta_{e}$ results from the first boundary condition, Eq. 29. This function presents a maximum, corresponding to critical conditions, which has been plotted versus $\Delta$ for several values of $N$ in Fig. 5. As was mentioned before, the critical value of $\Delta$ for $\theta_{w}<1$ is $1 / e$, resulting from the outer (Semenov) problem, whereas for $\theta_{w}>1$ the critical conditions are determined by the regions near the walls, where the reaction is enhanced by the higher temperature.

For large values of $N$ the radiation flux is small, and the critical Damköhler number never differs too much from the radiation-free value $1 / e$. For small values of $N$, the regions near the walls where the temperature decays become optically thick and the radiation behaves formally as thermal conduction. Both limiting cases are represented by dashed lines in Fig. 5.

\section{CONCLUSIONS}

An analysis has been carried out on the heterogeneous ignition of a coal dust cloud in air that accounts for heat conduction between the coal particles and the gas and radiative transfer between the particles. A large value of the gas/ particles thermal capacity ratio is considered, leading to a slow increase in the gas temperature 


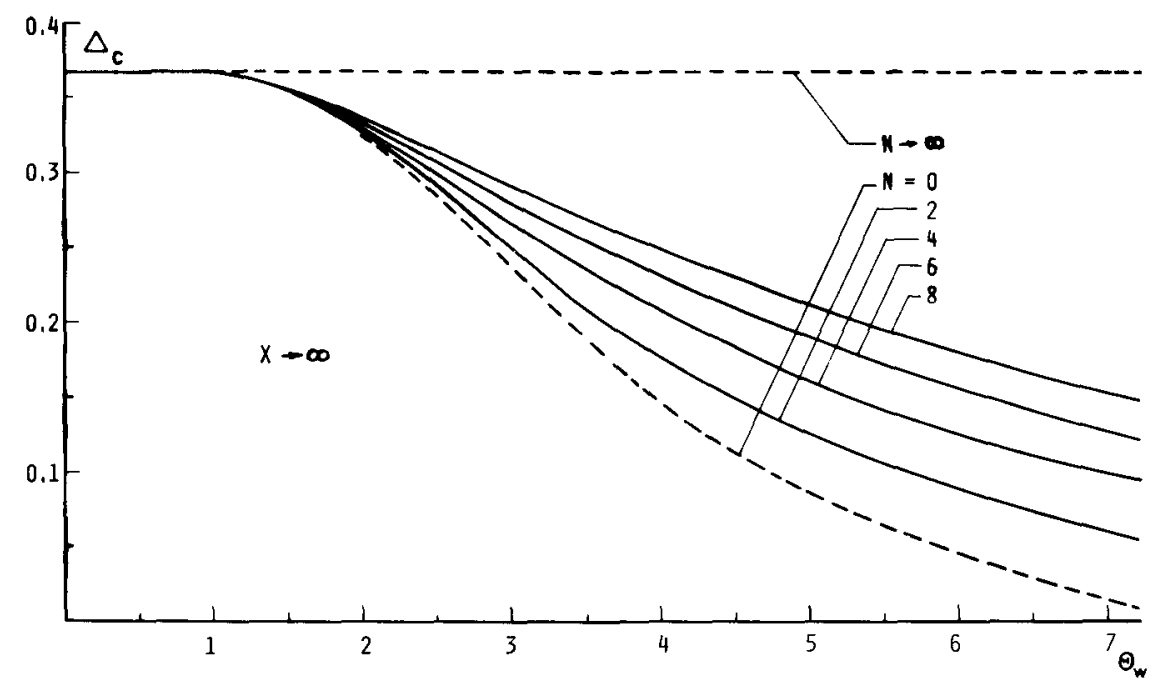

Fig. 5. Critical Damköhler number for a half-space bounded by a constant-temperature wall. Ignition occurs far from the wall when its temperature is smaller than that corresponding to critical conditions for an unbounded space $\left(\theta_{w}=1\right)$ and near the walls when its temperature is larger.

compared with the fast increase in the temperature of the particles, which adjusts itself to the conditions imposed by the gas and the walls.

When the temperature of the walls is lower than the initial temperature of the gas, the critical conditions for ignition are attained by the end of the particle heating, and the gas temperature has no time to change. In the ignition regime the heat released by the chemical reaction is appreciable only in a narrow optically thin region in the center of the cloud, where the temperature is maximum. The results presented correspond to very large values of the gas/particles thermal capacity ratio, when the temperature of the gas can be assumed to be constant even during the ignition process.

When the temperature of the walls is higher than the initial temperature of the gas, critical conditions for ignition occur by the end of the chemically frozen heating stage, when both temperatures are close to the wall temperature.

Special attention is paid to the case in which the temperatures of the gas and the walls bounding the system are close to each other, with the temperature difference on the order of $T_{g 0}{ }^{2} / T_{a}$. In this case the following results are obtained.

For large values of the conduction/radiation parameter the effect of the radiation can be neglected in the first approximation. Conduction between the particles and the gas is the only energy transfer mechanism left, and the classical Semenov ignition problem is recovered. In the opposite limit, $N \ll 1$, radiation transfer dominates over heat conduction. The temperature difference between the particles and the gas becomes unimportant, and the result is the problem analyzed by Joulin [11].

In between, for $N=O(1)$, ignition conditions depend on the temperature of the walls. If the walls are cold, that is, the wall temperature is lower than that corresponding to ignition in the homogeneous radiation-free case, the maximum heat release occurs at the middle of the cloud and a steady state with incipient chemical reaction exists only for small systems, with strong radiative losses toward the cold walls. For hot walls, the possibility arises of having a maximum particle temperature and maximum heat release near the walls; in this case ignition occurs for small systems, when the heat losses toward the center of the cloud become small or, which seems to be peculiar to radiative heat transfer, when the "apparent" conductivity vanishes at the walls. 
This behavior coexists with the behavior commented on earlier for cold walls if the conduction/ radiation parameter is smaller than a well-defined value, which depends on the wall temperature.

Asymptotic results are presented for both optically thin and optically thick media. From the results corresponding to optically thick media it can be seen that the limits $N \rightarrow 0$ and $X \rightarrow \infty$ do not commute.

The work of C.T. has been supported by the Foundation Alexander von Humboldt of the Federal Republic of Germany. F.J.H. and A.L. acknowledge partial support for this research from the Spanish CAICYT under project 2291083.

Zel'dovich, Ya. B., Barenblatt, G. I., Librovich, B. V., and Makhaviladze, G. M., The Mathematical Theory of Combustion and Explosions, Consultants Bureau, New York, 1985.

Ozisik, M. N., Radiative Transfer, Wiley, New York, 1973.

Cheng, P., AIAA J. 2(9):1662 (1964).

Essenhigh, R. H., and Csaba, J., Ninth Symposium (International) on Combustion, The Combustion Institute, Academic, New York, 1963, p. 111.

Bhaduri, D., and Bandyopadhyay, S., Combust. Flame 17:15 (1971).

Ozenova, G. E., and Stepanov, A. M., Combust. Explosion Shock Waves 9:543 (1973).

Krazinski, J. L., Buckius, R. O., and Krier, H., Prog. Energy Combust. Sci. 3:31 (1979).

Field, M. A., Gill, D. W., Morgan, D. B., and Hawksley, P. G. W., Combustion of Pulverized Coal, British Coal Utilization Research Association, Leatherhead, Surrey, England, 1967.

Khalil, H., Shultis, J. K., and Lester, T. W., J. Heat Transfer 105:288 (1983).

Khalil, H., Shultis, J. K., and Lester, T. W., Combust. Flame 53:149 (1983).

Joulin, G., Combust. Sci. Technol. 45 (1986).

Essenhigh, R. H., Sixteenth Symposium (International) on Combustion, The Combustion Institute, Academic, New York, 1976, p. 353.

Libby, P. A., and Blake, T. R., Combust. Flame 36:139 (1979).

Libby, P. A., Combust. Flame 38:285 (1980).

\section{APPENDIX A}

In this appendix we analyze the critical ignition transients in the realistic case of the gas/particles thermal capacity ratio, $I$, of order $\beta(I=n \beta)$ in the limit of optically thin media, $X \ll 1$. The corresponding nondimensional equations take the form

$$
\begin{aligned}
r^{3} \frac{\partial \theta_{s}}{\partial \tau}= & r\left(\theta_{g}-\theta_{s}\right) \frac{K}{e^{K}-1}-\frac{r^{2}}{N}\left(\theta_{s}{ }^{4}-\theta_{w}{ }^{4}\right) \\
& +\Delta r^{2} y_{s} \exp \left[\frac{\beta\left(\theta_{s}-1\right)}{\theta_{s}}\right]
\end{aligned}
$$

$I \frac{\partial\left(\rho \theta_{g}\right)}{\partial \tau}=-r\left(\theta_{g}-\theta_{s}\right) \frac{K}{e^{K}-1}$

$I \frac{\partial \rho}{\partial \tau}=\frac{C_{p} T_{m}}{Q} \Delta r^{2} y_{s} \exp \left[\frac{\beta\left(\theta_{s}-1\right)}{\theta_{s}}\right]$

$I \frac{\partial\left(\rho y_{g}\right)}{\partial \tau}=-\frac{4}{3 Y_{g 0}} \frac{C_{p} T_{m}}{Q} \Delta r^{2} y_{s} \exp \left[\frac{\beta\left(\theta_{s}-1\right)}{\theta_{s}}\right]$

$$
\begin{aligned}
3 \frac{C_{p}}{C_{s}} r \frac{\partial r}{\partial \tau}= & -\frac{C_{p} T_{m}}{Q} \Delta r^{2} y_{s} \\
& \times \exp \left[\frac{\beta\left(\theta_{s}-1\right)}{\theta_{s}}\right]=-K
\end{aligned}
$$

$\frac{\operatorname{Le} K}{\rho}=\ln \left[\frac{1+\left(3 Y_{g 0} / 4\right) y_{g}}{1+\left(3 Y_{g 0} / 4\right) y_{s}}\right], \quad K=\frac{m^{\prime \prime} R C_{p}}{k_{g}}$

where

$y_{s}=Y_{s} / Y_{g 0}, \quad y_{g}=Y_{g} / Y_{g 0}, \quad r=R / R_{0}$

$\mathrm{Le}=k_{g} / \rho_{g 0} C_{p} D_{g}, \quad \rho=\rho_{g} / \rho_{g 0}$.

All other parameters appearing in Eqs. A.1-A.6 are exactly the same as defined in the main text but with $T_{g 0}$ replaced by $T_{m}$, which is the equilibrium frozen temperature, given by the solution of the equation $k_{g}\left(T_{g 0}-T_{m}\right)=\sigma\left(T_{m}{ }^{4}-T_{w}{ }^{4}\right) R_{0}$. Equations A.1-A.6 have to be solved with the initial conditions $\theta_{s}=\theta_{s 0}, \theta_{g}=\theta_{g 0}, r=\rho=y_{g}$ $=1$. For $\bar{\tau} \rightarrow-\infty$, the solution for the first step 
(the frozen stage) is given up to terms of order $1 / \beta$ by

$$
\begin{aligned}
\theta_{s} \approx & 1+\frac{1}{\beta}\left\{C_{0} \exp \left[-\left(1+\frac{4}{N}\right) \bar{\tau}\right]\right. \\
& -\frac{\theta_{g 0}-1}{n(1+4 / N)} \bar{\tau}+\frac{D_{1}+\left(\theta_{g 0}-1\right) / n(1+4 / N)}{1+4 / N} \\
& \left.-\frac{\theta_{g 0}-1}{n(1+4 / N)} \tau^{*}\right\}+\cdots \\
\theta_{g} \approx & \theta_{g 0}+\frac{1}{\beta} \\
& \times\left[D_{1}-\frac{\left(\theta_{g 0}-1\right) \tau^{*}}{n}-\frac{\left(\theta_{g 0}-1\right) \bar{\tau}}{n}\right]+\cdots
\end{aligned}
$$

where

$$
\begin{gathered}
\bar{\tau}=\tau-\tau^{*}, \quad \tau^{*}=\frac{\ln \beta}{1+4 / N} \\
C_{0}=C_{0}\left(N, n, \theta_{w}, \theta_{s 0}\right), \\
D_{1}=D_{1}\left(N, n, \theta_{w}, \theta_{s 0}\right)
\end{gathered}
$$

The constants $C_{0}$ and $D_{1}$ can be obtained numerically by solving the problem in the frozen stage. In order to analyze the reactive stage, we introduce the following expansions:

$\theta_{s}=1+\frac{\psi_{s}}{\beta}+\frac{1}{\beta} \ln \frac{C_{s} T_{m} n \delta}{3 Q}+O\left(\frac{1}{\beta^{2}}\right)$

$\theta_{g}=\theta_{g 0}+\frac{\psi_{g}}{\beta}+O\left(\frac{1}{\beta^{2}}\right)$

$r=1+\frac{r_{1}}{\beta}+O\left(\frac{1}{\beta^{2}}\right) \quad y_{s}=1+\frac{y_{s 1}}{\beta}+O\left(\frac{1}{\beta^{2}}\right)$

$\rho=1+O\left(1 / \beta^{2}\right) \quad y_{g}=1+O\left(1 / \beta^{2}\right)$

where $\delta=\beta \Delta$ represents the appropriate Damköhler number. Introducing the relationships A.11 into Eq. A.1 and its time derivative, and eliminating the terms involving other dependent variables through the use of Eqs. A.2-A.6, we obtain

$\frac{d^{2} \psi_{s}}{d s^{2}}+\left(A-C e^{\psi_{s}}\right) \frac{d \psi_{s}}{d s}-e^{\psi_{s}}+1=0$

where

$$
\begin{aligned}
& A=\frac{(1+4 / N) n^{1 / 2}}{\left(\theta_{g 0}-1\right)^{1 / 2}}, \\
& C=\frac{3 C_{p}}{C_{s}} \frac{Q / C_{p} T_{m}-\left(1-\theta_{w}{ }^{4}\right) / 2 N}{n^{1 / 2}\left(1-\theta_{w}{ }^{4}\right)^{1 / 2}} N^{1 / 2},
\end{aligned}
$$

and

$s=\sqrt{\frac{n}{\theta_{g 0}-1}} \bar{\tau}$.

Equation A.12 is autonomous and can be rewritten as

$\frac{d \phi}{d u}=\frac{u-1-(A-C u) \phi}{u \phi}$

with

$\phi=\frac{d \psi_{s}}{d s}, \quad u=e^{\psi_{s}}$

For large negative values of the nondimensional time $s \rightarrow-\infty$, which correspond to $u \ll 1, \phi>$ 0 , the solution of A.14 is given, in first approximation, by

$$
\frac{u e^{\phi / A}}{(1+A \phi)^{1 / A^{2}}}=K
$$

where $K=K(A, C)$. In the phase-space $(u, \phi)$ there is only one trajectory that reaches the saddle point $(u, \phi)=(1,0)$, which matches with the frozen solution. This trajectory is associated with a critical value $K_{c}$ of the parameter $K$. For values $K$ $>K_{c}$ (supercritical behavior), the trajectory reaches asymptotically $\phi \approx C u$ with $(u, \phi)>1$. For $K<K_{c}$ (subcritical behavior), the trajectory ends in the nodal point $(u, \phi)=(0,-1 / A)$, which defines the quasi-steady slow-burning re- 
gime. Matching with the frozen stage requires that

$$
\begin{aligned}
K_{c}= & \frac{C_{s} T_{m}}{3 Q} \\
& \times \frac{n \delta_{c} \exp \left[D_{1} /(1+4 / N)\right]}{\left[\beta\left(\theta_{s 0}-1\right) / n(1+4 / N)\right]\left(-C_{0} A\right)^{1 / A^{2}}},
\end{aligned}
$$

which relates the critical value of $K$ with the critical Damköhler number $\delta_{c}$. Figures A.1-A.3 show the results obtained with different representative parameter sets. The characteristic behavior displayed at the right in the figures is associated with the condition $T_{s 0}=T_{m}$; the initial temperature of the particles becomes the same as the equilibrium temperature.

\section{APPENDIX B}

In this appendix a more detailed analysis is given for the limiting case of optically thick slabs $(X>$ 1) and small conduction/radiation parameter $(N \ll$ 1), in such a way that $\Lambda=\sqrt{N} X / 2=O(1)$.

Equations $23-25$ can be simplified to

$$
\frac{1}{\Lambda^{2}} \frac{d^{2} \theta}{d \zeta^{2}}=-\Delta e^{\Theta}+\theta
$$

$$
\zeta=0: \quad \frac{d \theta}{d \zeta}=0 ; \quad \zeta=1: \quad \theta=\theta_{w}
$$

where $\xi=\xi / X$. Here the second derivative term in Eq. 24 and the terms proportional to the radiation flux $\Phi$ in the boundary condition of Eq. 25 have been neglected. Equation B.1 can be

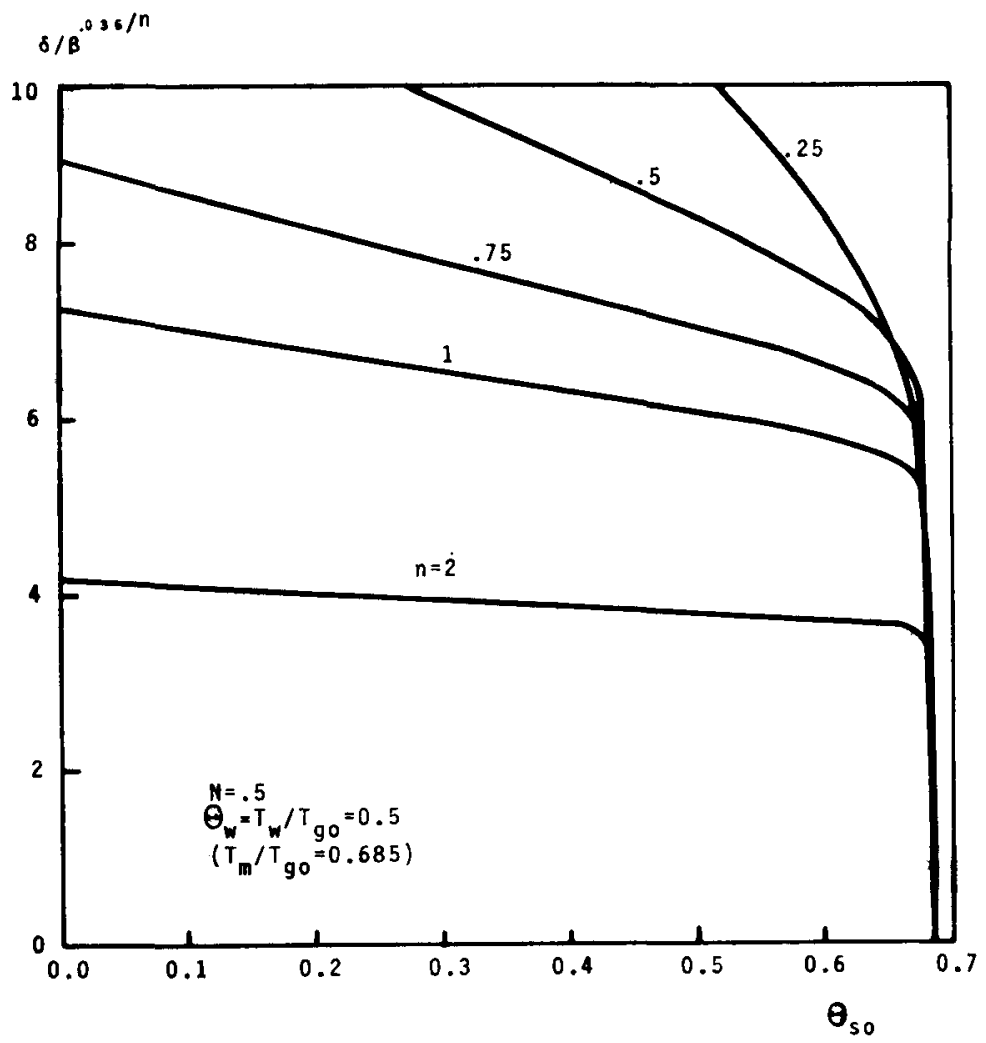

Fig. A.1. Critical conditions for ignition for $N=0.5, \theta_{w}=T_{w} / T_{g 0}=0.5\left(T_{m} / T_{g 0}=\right.$ 0.685 ) and various values of $n$. 


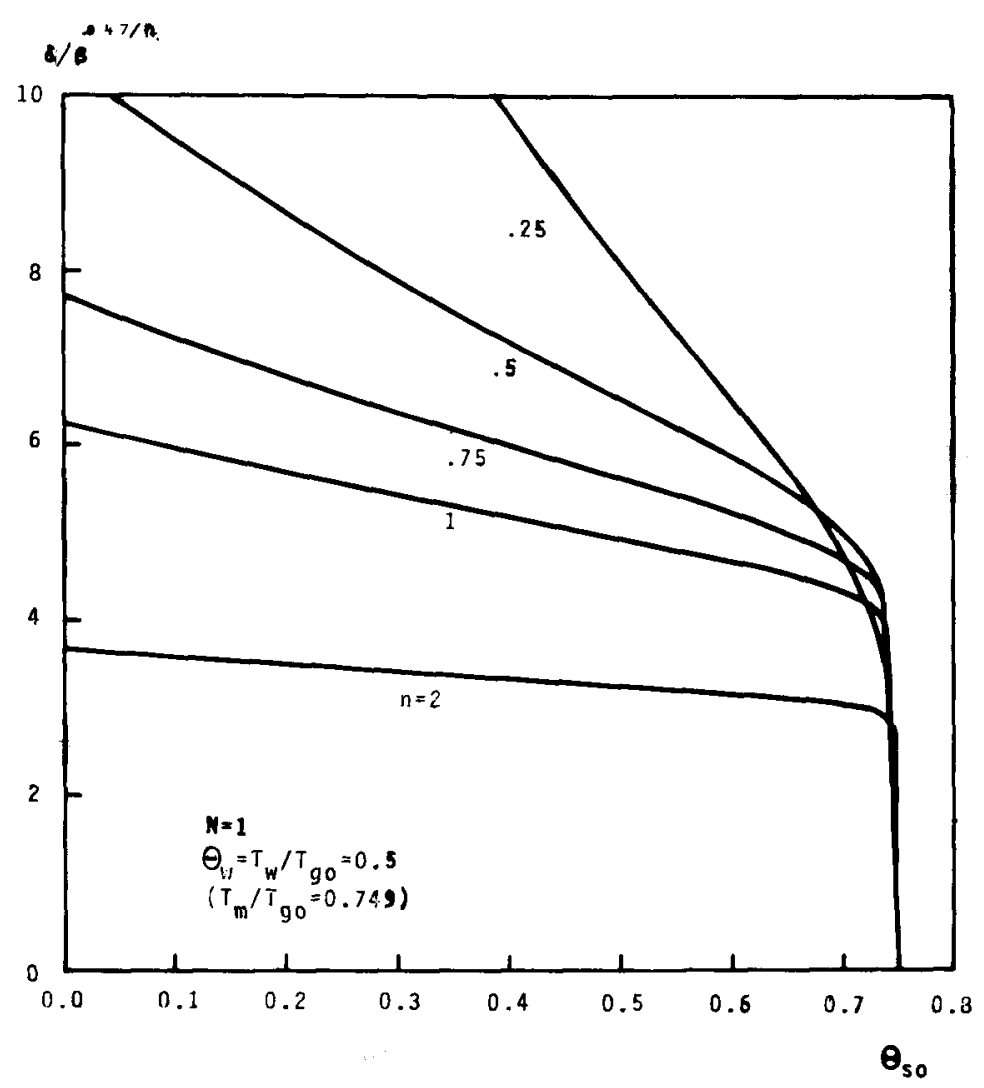

Fig. A.2. Critical conditions for ignition for $N=1, \theta_{w}=T_{w} / T_{g 0}=0.5\left(T_{m} / T_{g 0}=0.749\right)$ and various values of $n$.

rewritten in the form

$\frac{1}{\Lambda} \frac{d \Theta}{d \zeta}=P$

$P^{2}+2 \Delta e^{\theta}-\theta^{2}-2 \Delta e^{\theta_{0}}+\theta_{0}^{2}=0$

showing the phase-plane structure of Fig. B.1.

If $\Delta$ is larger than the Semenov critical value 1/ $e$ for the particles in the radiation-free case, a typical trajectory in the phase plane begins at $p=$ $0, \theta=\theta_{0}>\theta_{w}$ and ends at a negative value $p_{1}$ of $p$ at $\theta=\theta_{w}$. The nondimensional size $\Lambda$ of the slab is given in terms of $\Theta_{0}$ by

$\Lambda=\int_{\theta_{w}}^{\theta_{0}} \frac{d \theta}{\left[2 \Delta\left(e^{\theta_{0}}-e^{\theta}\right)+\theta^{2}-\theta_{0}^{2}\right]^{1 / 2}}$

This leads to the limit $\Lambda \rightarrow 0$ for both $\theta_{0} \rightarrow \theta_{w}$ and $\theta_{0} \rightarrow \infty$, so that $\Lambda$ has a maximum $\Lambda_{c}$ at some intermediate value of $\theta_{0}$. Two solutions exist, therefore, for $\Delta>1 / e$, with higher temperatures in the center of the slab than at the wall if $\Lambda<$ $\Lambda_{c}\left(\Delta, \theta_{w}\right)$. There are no solutions with particle temperatures $\theta<\theta_{w}$.

If $\Delta<1 / e$, a number of possibilities arise depending on the relative values of $\theta_{w}$ and the temperatures $\theta_{*}>\theta_{+}>\theta_{-}$. Here $\theta_{*}$ is the maximum value of $\Theta$ in the phase-plane trajectory that goes through $\theta_{-} . \theta_{*}$ satisfies the equation

$2 \Delta\left(e^{\theta_{*}}-e^{\theta_{-}}\right)-\left(\theta_{*}^{2}-\theta_{-}^{2}\right)=0$

while $\theta_{+}$and $\theta_{-}$are the two roots of

$\Delta e^{\theta}-\theta=0$

If $\theta_{w}>\theta_{*}$, the only type of solutions that exist are those shown in Fig. B.2a, similar to the ones of 


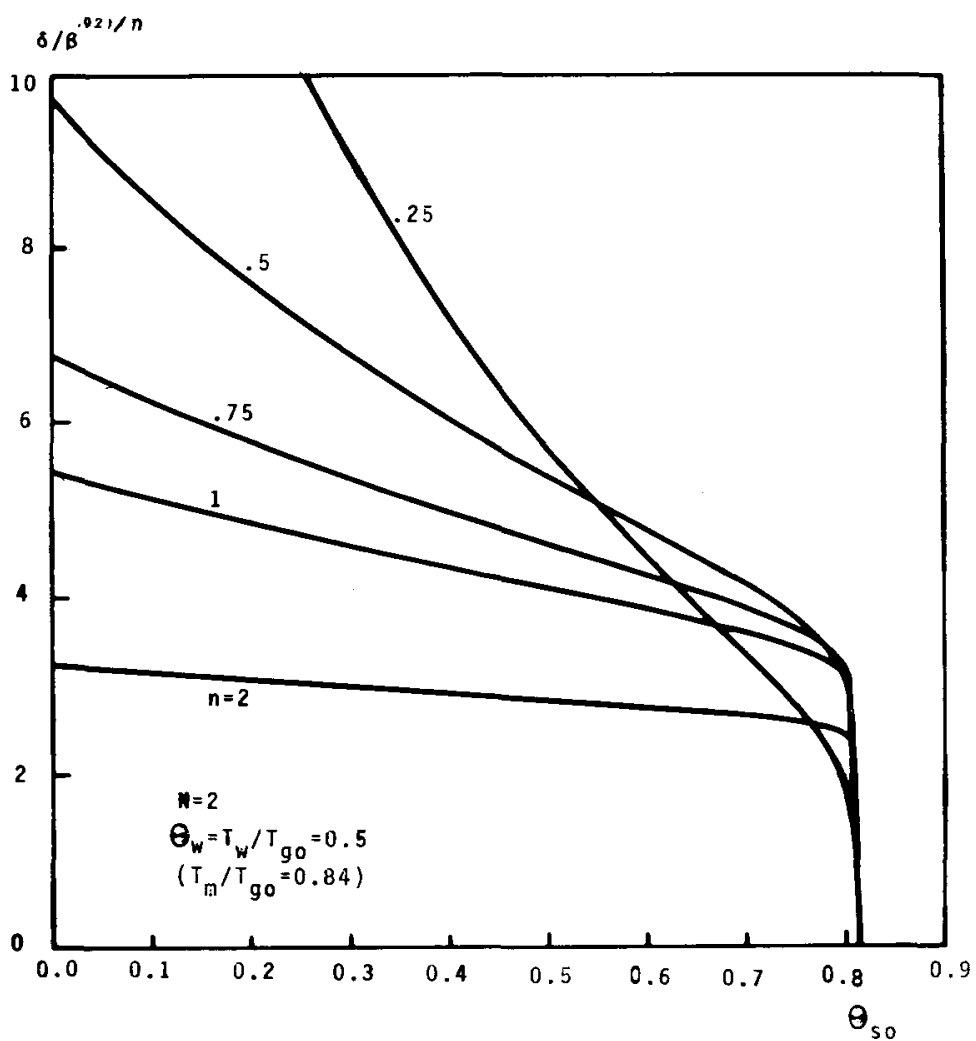

Fig. A.3. Critical conditions for ignition for $N=2, \theta_{w}=T_{w} / T_{g 0}=0.5\left(T_{m} / T_{g 0}=0.84\right)$ and various values of $n$.

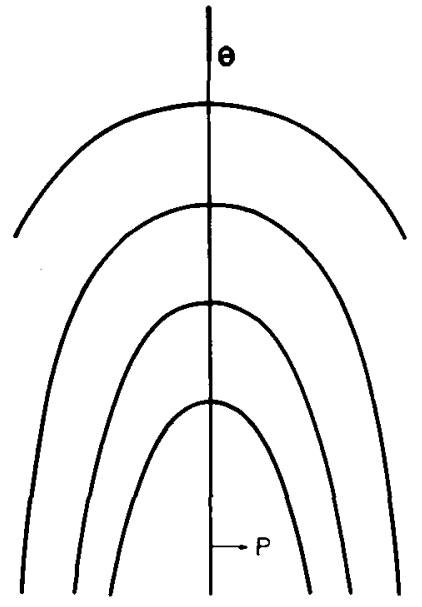

$\Delta=1$

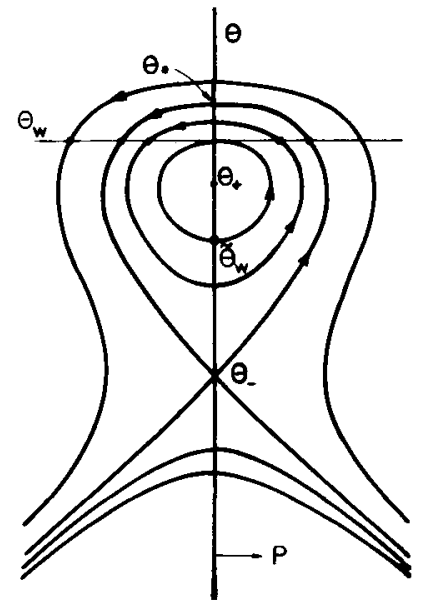

$\Delta=.3$

Fig. B.1. Phase portraits for Eqs. B.3 and B.4 for two values of $\Delta$, larger and smaller than $1 /$ $e$. The arrows in the second plot show the two kinds of phase trajectories possible for a value $\theta_{+}<\theta_{w}<\theta_{*}$. 

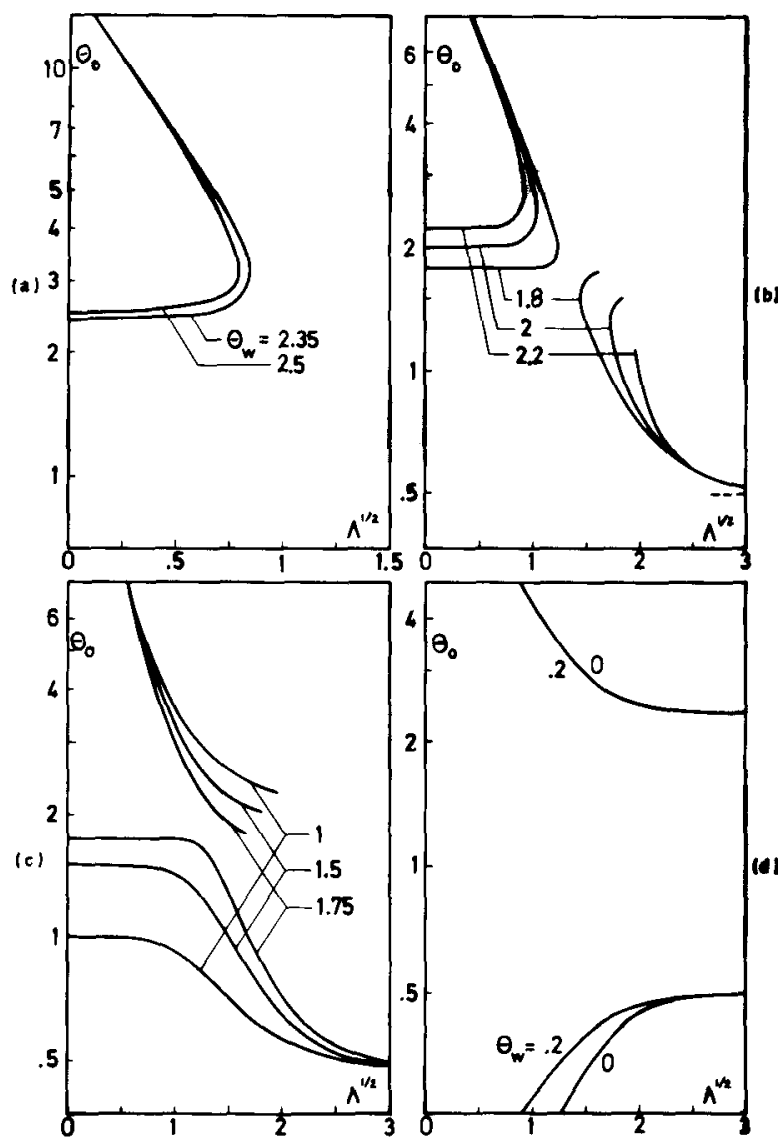

Fig. B.2. Temperature at the center of the slab as a function of $\Lambda^{1 / 2}$ for $\Delta=0.3$ and several values of $\theta_{w}$ in the different ranges (a) $\theta_{*}<\theta_{w}$; (b) $\theta_{+}<\theta_{w}<\theta_{*}$; (c) $\theta_{-}<\theta_{w}<\theta_{+}$; (d) $\theta_{w}<\theta_{-}$.

the case $\Delta>1 / e$; they exist for $\Lambda<\Lambda_{c}\left(\Delta, \theta_{w}\right)$. On the other hand, if $\theta_{+}<\theta_{w}<\theta_{*}$, then, besides the old type of solutions with $p<0$ and $\theta$ $>\theta_{w}$, a new type of solutions, with $p>0$, exists, with the phase-plane trajectories shown on the right in Fig. B. Ib. In these new solutions, $\theta<$ $\theta_{w}$ everywhere. The particle temperature $\Theta_{0}$ at the center of the slab is $\theta_{-}<\theta_{0}<\tilde{\theta}_{w}$, where $\tilde{\theta}_{w}<$ $\theta_{w}$ is the middle root of the equation

$$
2 \Delta\left(e^{\tilde{\theta}_{w}}\right)-\left(\tilde{\Theta}_{w}{ }^{2}-\theta_{w}{ }^{2}\right)=0
$$

The particles receive heat by radiation in the center of the slab and lose it close to the walls. These solutions exist only for $\Lambda$ larger than a minimum value $\Lambda_{\min }\left(\Delta, \theta_{w}\right) \leqslant \Lambda_{l}\left(\Delta, \theta_{w}\right)$, where $\Lambda_{l}$ is the length associated with $\theta_{0}=\tilde{\theta}_{w}$. The trajectories in the phase plane lie in the domain $\theta_{-}$ $<\theta<\theta_{w}$ bounded by those originating at $\theta_{-}$ and $\tilde{\theta}_{w}$. The solution associated with $\theta_{-}$corresponds to infinite $\Lambda$. Some examples of both kinds of solutions for $\theta_{+}<\theta_{w}<\theta_{*}$ are shown in Fig. B.2b.

If $\theta_{-}<\theta_{w}<\theta_{+}$, solutions with $p>0$ and $\theta_{-}<\theta<\theta_{w}$ exist for all values of $\Lambda$, while the solutions with $p<0$ are restricted to values of $\theta_{0}$ $>\tilde{\Theta}_{w}$, where $\tilde{\Theta}_{w}$ is the upper root of Eq. A.8. The relation between $\Lambda$ and $\theta_{0}$ is of the type shown in Fig. B.2c.

Finally, for $\theta_{w}<\theta_{-}$, two types of solutions exist with $p<0$, corresponding to $\theta_{w}<\theta_{0}<$ $\theta_{-}$and $\theta_{0}>\theta_{*}$, and there are no solutions with $\theta_{0}$ in the intermediate range; see Fig. B.2d. 